\title{
A Mesoscopic Field Evaporation Model
}

\author{
Constantinos Hatzoglou ${ }^{1}$ and Francois Vurpillot ${ }^{1 *}$ \\ 1. Normandie Université, UNIROUEN, INSA Rouen, CNRS, Groupe de Physique des Matériaux, \\ Rouen, France \\ * Corresponding author: francois.vurpillot@univ-rouen.fr
}

To date, it is no longer necessary to demonstrate the contribution and the usefulness of field evaporation simulation to understand and to interpret atom probe tomography (APT) analyses [1, 2]. In addition, recently, simulation has been also used to perform APT data reconstruction [3]. There are many commonly used simulation models, from sub-atomic scale [4,5] up to atomic scale [2], according to the specific studied phenomena. Nevertheless, it appears today a need to have simulation models at lower scale (i.e. mesoscopic scale), such as level set methods [6]. Even if, these models show qualitative agreement with experimental data in 2D simulation [7], it is no completely the case in 3D, where some improvements remain to be performed [8]. In this paper, a new field evaporation simulation in 3D at the mesoscopic scale is presented. The aptitude of this new approach to reproduce APT experimental observations, while maintaining low computational and memory resources, is demonstrated through the simulation of analyses of complex microstructures under laser irradiation.

In this model, the surface is defined by an assembly of meshes, which amounts to perform a surface triangulation (figure 1.a). This step introduced the mesoscopic approach of the simulation. Furthermore, it is realized in such ways that each mesh has the same surface (editable simulation parameters), at the initial step of the simulation. It is then possible to adjust the scale of the simulation to features of interest. To reflect the experimental evaporation rate, all the meshes vertices are continuously displaced. For each vertex, the displacement direction is estimated using the normal direction of its surrounding meshes and the amplitude, using the evaporation probability, also from its surrounding meshes $\left(P_{j}\right)$, defined as follow:

$$
P_{j}=\exp \left(\frac{Q_{0}}{k_{B} T_{j}}\left(1-\frac{F_{j}}{F_{E V, j}}\right)\right)
$$

with $Q_{0}$ the energy barrier without any external field, $k_{B}$ the Boltzmann constant, $T_{j}$ the temperature, $F_{j}$ the electrical field on the mesh $j$ and $F_{E V, j}$ its evaporation field. The temperature of each meshes $\left(T_{j}\right)$ can be set as constant (between $20-80 \mathrm{~K}$ for example as experimentally used) to reproduce evaporation assisted by electrical pulses, but also can be set spatially dependant to reflect the laser assisted evaporation process. The maximal temperature distribution at the sample surface induced by the laser interaction is estimated using the software Lumerical. The simulated microstructure is defined through the evaporation field $\left(F_{E V, j}\right)$ of each phase. For a single phase material, all the meshes have the same evaporation field. For example, in a case of vertical layers (figure 1.a), a higher (figure 1.b) or a lower (figure 1.c) evaporation field than the surrounding matrix can be assigned. The required electrical field $F_{j}$ on each mesh is calculated from the charge distribution at the sample surface at each step of surface modification, using the Robin equation (similar to the atomistic Robin-Rolland field evaporation model) [9].

This model reproduces quantitatively experimental APT analysis observations and measurements. Moreover, it provides at each step of the simulation important information (morphology, charge 
distribution, electrical field ...) than can be used then to compute ion trajectory up to the detection system.

\section{References:}

[1] Hatzoglou et al., Journal of Nuclear Materials 505 (2018), p. 240.

[2] Vurpillot et al., Ultramicroscopy 159 (2015), p. 202.

[3] Beinke et al., Ultramicroscopy 165 (2016), p. 34.

[4] Karahka et al., Ultramicroscopy 132 (2013), p. 54.

[5] Zanuttini et al., Phy. Rev. A. 95 (2017).

[6] Haley et al., Microscopy and Microanalysis 19 (2013), p. 1709.

[7] Xu et al., Computer Physics Communications 189 (2015), p. 106.

[8] Haley et al., Materials characterization 146 (2018), p. 299.

[9] Rolland et al., Microscopy and Microanalysis 21 (2015), p. 1649.

(b)

(a)

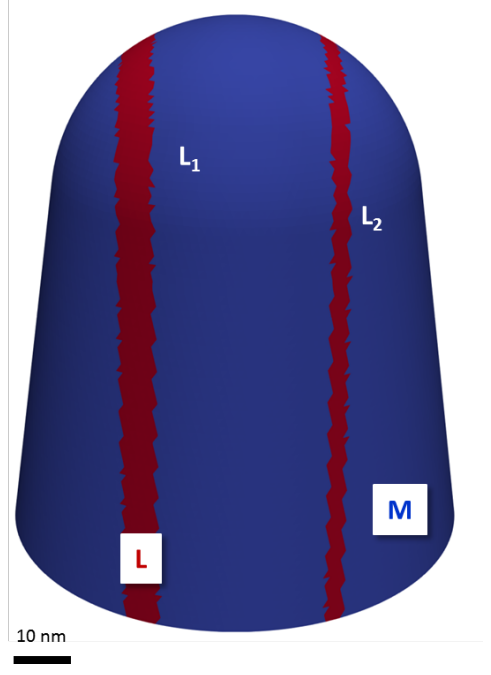

$10 \mathrm{~nm}$
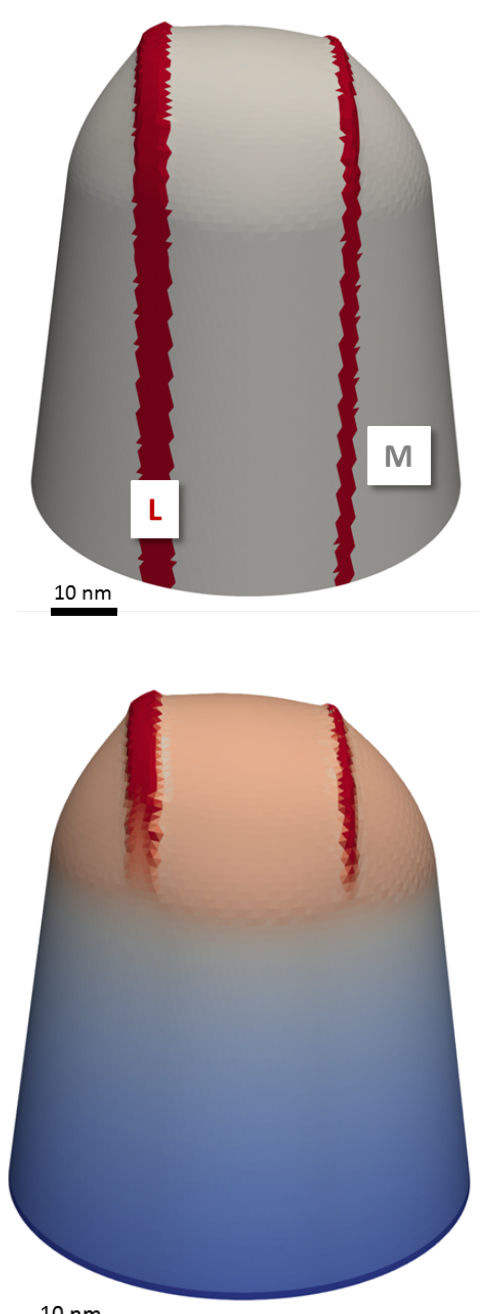

$10 \mathrm{~nm}$ (c)
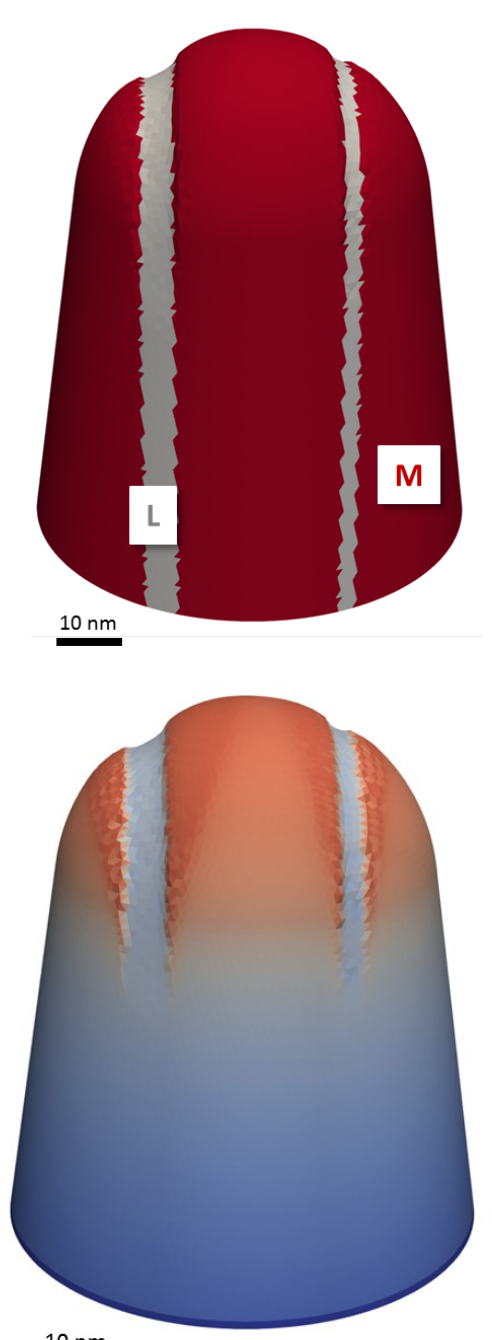

$10 \mathrm{~nm}$

Figure 1. (a) Sample at mesoscopic scale simulation composed of two vertical layers (thickness of $6 \mathrm{~nm}$ on the left part and $3 \mathrm{~nm}$ on the other part) before field evaporation: radius of curvature of $35 \mathrm{~nm}$, half shank angle of $9^{\circ}$ and a triangle are of $0.5 \mathrm{~nm}^{2}$. The upper part is the sample morphology and the lower part is the electrical field at the surface during the evaporation, if the layers have (b) a higher or (c) a lower evaporation field than the surrounding matrix. 